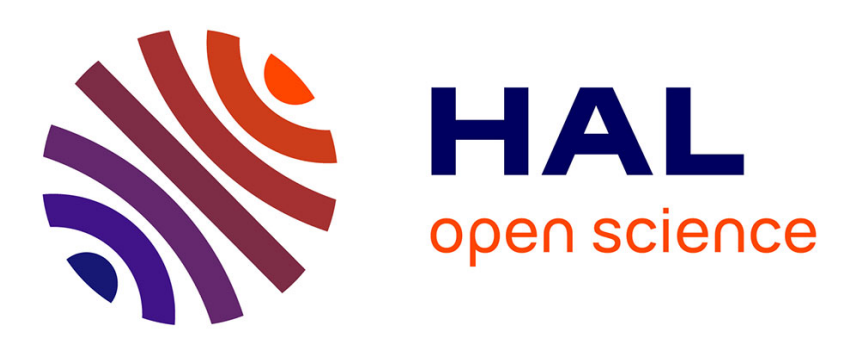

\title{
Defining an equilibrium state in global full-f gyrokinetic models
}

Guilhem Dif-Pradalier, Virginie Grandgirard, Yanick Sarazin, Xavier Garbet, Philippe Ghendrih

\section{- To cite this version:}

Guilhem Dif-Pradalier, Virginie Grandgirard, Yanick Sarazin, Xavier Garbet, Philippe Ghendrih. Defining an equilibrium state in global full-f gyrokinetic models. Communications in Nonlinear Science and Numerical Simulation, 2008, 13, pp.65 - 71. 10.1016/j.cnsns.2007.05.004 . cea-01468358

\section{HAL Id: cea-01468358 https://hal-cea.archives-ouvertes.fr/cea-01468358}

Submitted on 15 Feb 2017

HAL is a multi-disciplinary open access archive for the deposit and dissemination of scientific research documents, whether they are published or not. The documents may come from teaching and research institutions in France or abroad, or from public or private research centers.
L'archive ouverte pluridisciplinaire HAL, est destinée au dépôt et à la diffusion de documents scientifiques de niveau recherche, publiés ou non, émanant des établissements d'enseignement et de recherche français ou étrangers, des laboratoires publics ou privés. 


\title{
Defining an equilibrium state in global full-f gyrokinetic models
}

\author{
G. Dif-Pradalier *, V. Grandgirard, Y. Sarazin, X. Garbet, Ph. Ghendrih \\ Association EURATOM-CEA, CEAIDSMIDRFC, Bât 513, CEA Cadarache, 13108 St Paul-lez-Durance, France
}

Available online 25 May 2007

\begin{abstract}
This paper tackles the delicate choice of the initial distribution function in full- $f$ gyrokinetic codes such as GysELA 5D, aiming at predicting the turbulent transport level in low collisional tokamak plasmas. It is found, both analytically and numerically, that a Maxwellian distribution function with constant profiles on magnetic flux surfaces leads to the fast generation of a large scale electric field. Such a field opposes the up-down charge separation governed by the inhomogeneity of the equilibrium magnetic field. If large enough, the shearing rate induced by the resulting poloidal $\mathbf{E} \times \mathbf{B}$ velocity could efficiently reduce the plasma micro-instabilities which account for the development of the turbulence. Starting in the ab initio code GYSELA 5D from an equilibrium distribution function depending on motion invariants only is shown to cure such a problem. In this case, charge separation is counter-balanced by parallel flow, and the standard fluid force balance is recovered. (C) 2007 Elsevier B.V. All rights reserved.
\end{abstract}

PACS: $52.30 . \mathrm{Gz} ; 52.65 . \mathrm{Tt}$

Keywords: Tokamak plasma; Nonlinear gyrokinetic simulations; Collisionless equilibrium definition

\section{Introduction}

In tokamak plasmas, the energy confinement time is largely governed by the magnitude of the turbulent transport. Nonlinear fluid simulations have been recently reported to systematically overestimate such a transport [1]. Gyrokinetic models look appropriate to study such low collisional plasmas. However, they suffer from the large numerical resources they require, inherent to the intrinsic complexity of a five-dimensional phase space. In this framework, most present gyrokinetic codes deal with fluctuations only, implicitly assuming a scale separation with the equilibrium. This assumption potentially conflicts with large scale fast transport events, sometimes reported both in experiments [2] and in flux driven numerical simulations [3-5].

Conversely, the GYrokinetic SEmi-LAgrangian Gysela code [6] treats the entire ion distribution function in an axi-circular toroidal geometry. Such a full- $f$ code faces the delicate problem of solving the gyrokinetic equilibrium in a collisionless tokamak plasma. This is a crucial issue in those turbulence simulations where the linear and nonlinear regimes of the instability are let to evolve from an initial unstable equilibrium state. Especially, the first section shows that any equilibrium state at vanishing electric field requires a finite parallel

\footnotetext{
* Corresponding author. Tel.: +334422540 25; fax: +33442256233.

E-mail address: guilhem.dif-pradalier@cea.fr (G. Dif-Pradalier).
} 
flow in order to compensate the vertical charge separation born of the inhomogeneity of the magnetic field. If present, this large scale electric potential may increase the effective threshold of the instability, and potentially quench the turbulence if its magnitude is large enough. Here, charge separation is to be understood in terms of plasma polarisation, since $\left(\delta n_{e}-\delta n_{i}\right) / n_{0} \lll 1$. Section 3 provides a natural way to account for the parallel flow in GYSELA - thus preventing the generation of any equilibrium electric potential - by considering distribution functions depending on the motion invariants only. Finally, the consistency of the retained equilibrium with the force balance equation is discussed in Section 4.

\section{Non-canonical initial distribution functions generate poloidal sheared flows}

The onset of turbulence and its characteristics both depend on the properties of the equilibrium it develops onto. Such an intricate relationship is of prominent importance in those codes where no scale separation between equilibrium and fluctuations is assumed. This is especially true for the gyrokinetic code GYSELA 5D, which self-consistently solves the gyrokinetic and the quasi-neutrality equations for the entire ion distribution function in a simplified toroidal geometry $[7,8]$. In such a full- $f$ code, the system is initialised with an equilibrium distribution function plus a bath of small amplitude modes. These small perturbations eventually grow and nonlinearly saturate, provided the initial equilibrium is above the linear stability threshold. Conversely, if the initial distribution function is not an equilibrium function, the system will first evolve towards an equilibrium. The consequences are twofold: the time evolution of the initial perturbations will be hard to discriminate from that of the search of an equilibrium, and the equilibrium found by the system, if any, may turn out to be stable with regard to perturbations.

In that respect, it is interesting to consider the following Maxwellian initial distribution function:

$$
f_{\mathrm{M}}=\frac{n(r)}{(2 \pi T(r) / m)^{3 / 2}} \mathrm{e}^{-E / T(r)}
$$

where $n$ and $T$ are the radial profiles of density and temperature and $E=m v_{\|}^{2} / 2+\mu B . f_{\mathrm{M}}$ is constant on a magnetic surface labelled by the radial coordinate $r$. We shall call this initialisation non-canonical. As will be shown in this section, such an initial state does not constitute an equilibrium of the system solved by GySELA 5D at vanishing electric field. Indeed, a radial electric field will be shown to develop on short time scales. The resulting sheared poloidal flow can then possibly prevent the onset of turbulence, consistently with theoretical predictions [9-11].

Especially, let us show analytically that any initial distribution function similar to the one defined by Eq. (1) leads to the development of a non-vanishing electric field in GysELA 5D. The fundamental reason is that such a non-canonical $f$ does not carry any parallel flow that would ensure the compensation of the vertical charge separation governed by the curvature and $\operatorname{grad}-B$ drifts in a toroidal geometry. The fluid analogous of such a physics is the fact that the divergence of the diamagnetic velocity has to be balanced by the divergence of the parallel flow [12]. The time evolution of the guiding-centre 5D distribution function $f\left(r, \theta, \varphi, v_{\|}, \mu\right)$ solved in GYSELA - namely the gyrokinetic equation - can be found in Grandgirard et al. [7]. Let us look for equilibrium solutions at vanishing electric field and of the following general form: $f_{\text {eq }}=g_{\mu}\left(r, \theta, v_{\|}\right) \mathrm{e}^{-E / T(r)}$, where $g_{\mu}$ is a given function of $r, \theta$ and $v_{\|}$parametrised by $\mu$. The gyrokinetic equation then reads

$$
v_{g}\left\{g_{\mu} \mu B \frac{\partial_{r} T}{T^{2}} \sin \theta+g_{\mu} v_{\|}^{2} m \frac{\partial_{r} T}{2 T^{2}} \sin \theta+\partial_{r} g_{\mu} \sin \theta+\partial_{\theta} g_{\mu} \frac{\cos \theta}{r}\right\}+\partial_{v_{\|}} g_{\mu} \frac{\mu B r \sin \theta}{m q R^{2}}-\partial_{\theta} g_{\mu} \frac{v_{\|}}{q R}=0
$$

where $\mathbf{v}_{g}=\frac{m v_{\|}^{2}+\mu B}{e B} \frac{\mathbf{B} \times \mathbf{\nabla} B}{B^{2}}=-\frac{m v_{\|}^{2}+\mu B}{e R_{0} B_{0}}\left(\sin \theta \hat{\mathbf{e}}_{r}+\cos \theta \hat{\mathbf{e}}_{\theta}\right)$ is the curvature and grad- $B$ drifts in the low $\beta$ approximation, $v_{g}$ its modulus, $\mathbf{B}=B\left(\hat{\mathbf{e}}_{r}+r / q R_{0} \hat{\mathbf{e}}_{\theta}\right)$ is the magnetic field, $B=B_{0} R_{0} / R, R=R_{0}+r \cos \theta$ the major radius and $q$ the safety factor. We also concentrate on an axisymmetric equilibrium distribution $\partial_{\varphi} \equiv 0$. We intend to show that $g_{\mu}$ cannot be an even function of $v_{\|}$and must be such that any equilibrium at vanishing electric field requires a finite parallel flow. To proceed, let us assume by contradiction an equilibrium distribution function can exist, with a fixed even parity in $v_{\|}$, such that $g_{\mu}\left(r, \theta, v_{\|}^{2}\right)=\sum_{n=0}^{\infty} a_{n}(r, \theta) v_{\|}^{2 n}$. Projecting Eq. (2) on the $\left\{v_{\|}^{2 p}, v_{\|}^{2 p+1}\right\}(p \in \mathbb{N})$ basis allows one to separate the odd and even parities in $v_{\|}$: 


$$
\begin{aligned}
& \mu B \frac{\partial_{r} T}{T^{2}} \sin \theta\left\langle g_{\mu} v_{g} v_{\|}^{2 p}\right\rangle+m \sin \theta \frac{\partial_{r} T}{2 T^{2}}\left\langle g_{\mu} v_{g} v_{\|}^{2 p+2}\right\rangle+\sin \theta\left\langle\partial_{r} g_{\mu} v_{g} v_{\|}^{2 p}\right\rangle+\frac{\cos \theta}{r}\left\langle\partial_{\theta} g_{\mu} v_{g} v_{\|}^{2 p}\right\rangle=0 \\
& \frac{\mu B r \sin \theta}{m R}\left\langle\partial_{v_{\|}} g_{\mu} v_{\|}^{2 p+1}\right\rangle-\left\langle\partial_{\theta} g_{\mu} v_{\|}^{2 p+2}\right\rangle=0
\end{aligned}
$$

where the brackets stand for $\langle\mathscr{G}\rangle \equiv \int_{-\infty}^{+\infty} \mathrm{d} v_{\|} \mathscr{G} \exp \left(-m v_{\|}^{2} / 2 T_{0}\right)$. Using the expression of $g_{\mu}$, Eq. (3b) reads as follows:

$$
\sum_{n=0}^{\infty}\left(\frac{2 T_{0}}{m}\right)^{n} \Gamma\left(n+p+\frac{1}{2}\right)\left\{n a_{n} \frac{2}{m} \frac{\mu B r \sin \theta}{R}-\partial_{\theta} a_{n-1}\right\}=0
$$

with $\Gamma(n) \equiv \int_{0}^{\infty} \mathrm{d} t t^{n-1} \mathrm{e}^{-t}$. Since Eq. (4) must remain valid for all integer $p$, the following relationship holds, for all integers $n \geqslant 1$ :

$$
a_{n}=\frac{m R}{2 \mu B r \sin \theta} \frac{\partial_{\theta} a_{n-1}}{n}
$$

Injecting such a relationship in Eq. (3a) yields for the $a_{0}$ term:

$$
\left[B, a_{0}\right]=a_{0} \frac{\mu B \partial_{\theta} B}{r} \frac{\partial_{r} T}{T^{2}}
$$

where $[g, h] \equiv \frac{1}{r}\left(\partial_{r} g \partial_{\theta} h-\partial_{r} h \partial_{\theta} g\right)$ is a Poisson bracket. Eq. (6) implies that $\frac{1}{\sin \theta}\left[B, a_{0}^{2}\right]=\frac{2 \mu B^{2}}{R} \frac{\partial_{r} T}{T^{2}} a_{0}^{2}$. Integrated over $r$ and $\theta$, the latter equation can be recast as follows:

$$
\int \mathrm{d} r \mathrm{~d} \theta \frac{a_{0}^{2}}{R^{2}}\left\{\frac{1}{r \sin ^{2} \theta}-2 \mu \frac{\partial_{r} T}{T^{2}}\right\}=\int \mathrm{d} \theta\left[a_{0}^{2} \frac{B}{R^{2}}\right]_{r_{\min }}^{r_{\max }}
$$

The left-hand side integrand is strictly positive. The right-hand side integrand depends on $\theta$ only. Therefore, when differentiating Eq. (7) with respect to $r$, and for any $a_{0}$ and $T$ regular enough, the only physical solution is for $a_{0}$ identically equal to zero. Eq. (5) then implies that all the other coefficients $a_{n}$ are also identically zero. Thus, with a vanishing electric potential, the only even solution in $v_{\|}$of the gyrokinetic equation is null everywhere. In other words, any possible equilibrium solution that is even in $v_{\|}$- which is especially the case if $g_{\mu}$ is independent of $v_{\|}$- is characterised by a non-vanishing electric field. Alternatively, equilibrium solutions at zero electric field are asymmetric in $v_{\|}$, such that there exists a finite parallel flow analogous to the PfirschSchlüter current. Notice that this flow may be vanishing when averaged on a flux surface, similarly to the Pfirsch-Schlüter current.

As will be shown in the following section, such a property is intrinsically fulfilled when properly considering an equilibrium distribution function $[13,14]$.

\section{Canonical equilibrium in GrSELA 5D}

Any arbitrary function of the motion invariants - namely the adiabatic invariant $\mu=m v_{\perp}^{2} / 2 B$, the total energy $\mathscr{H}=\frac{1}{2} m v_{\|}^{2}+\mu B+e \phi$ and the toroidal kinetic momentum $P_{\varphi}=m R v_{\varphi}+e \psi$, with $\psi$ the poloidal flux represents a stationary equilibrium in the collisionless gyrokinetic code GYSELA 5D. Let $f_{\text {eq }}$ be the so-called canonical equilibrium, defined as a kind of modified Maxwellian:

$$
f_{\text {eq }}=\frac{n(\bar{r})}{(2 \pi T(\bar{r}) / m)^{3 / 2}} \mathrm{e}^{-\mathscr{H} / T(\bar{r})} \mathrm{e}^{+e \phi(\bar{r}) / T(\bar{r})}
$$

where $\bar{r}$ stands for an effective radial coordinate derived from the third invariant: $\bar{r}=r_{0}-\frac{q_{0}}{r_{0} e B_{0}} P_{\varphi}+h(\mu, \mathscr{H})$, such that $f_{\text {eq }}$ is a function of the motion invariants only (the quantities with a label 0 are defined at half-radius of the simulation box and $h$ is an arbitrary function of the motion invariants $\mu$ and $\mathscr{H}$ ). The density $n$ and temperature $T$ are no longer radial profiles, but functions of the motion invariant $P_{\varphi}$. Since the parallel 
dynamics is crucial to equilibrium, $\bar{r}$ is written in terms of $P_{\varphi}$ so as to bear this dependence. Following [14], a good choice appears to be

$$
\bar{r}=r_{0}-\frac{q_{0}}{r_{0}}\left[\psi(r)-\psi\left(r_{0}\right)\right]-\frac{m q_{0}}{e B_{0} r_{0}}\left[R v_{\|}-R_{0} \bar{v}_{\|}\right]
$$

where $\psi(r)=\int_{0}^{r} \frac{r^{\prime} \mathrm{d} r^{\prime}}{q}$. Also, the last term in Eq. (9) is by definition a motion invariant, which will be assumed to scale like $\rho^{\star}$, the ratio of the ion gyroradius $\rho_{i}$ over the minor radius:

$$
\bar{v}_{\|}=\operatorname{Sign}\left(v_{\|}\right) \sqrt{\frac{2}{m}} \sqrt{E-\mu B_{\max }} \mathscr{Y}\left(E-\mu B_{\max }\right)
$$

with $\mathscr{Y}$ the Heaviside function and $B_{\max }$ the maximum of the magnetic field on the whole simulation box. It is worth noticing that, due to the explicit dependence in $v_{\|}$of the invariant $\bar{r}$, the canonical $f_{\text {eq }}$ exhibits an asymmetry in $v_{\|}$. Such a property allows for the compensation of the vertical charge separation by a finite parallel flow, as discussed in the previous section. This justifies a posteriori the use of the invariant $\bar{r}$ as an effective radial coordinate. So as to minimise the parallel velocity, $\bar{v}_{\|}$in Eq. (9) is chosen such that $\left(R v_{\|}-R_{0} \bar{v}_{\|}\right)$scales like $\rho^{\star}$. It is important to notice that even though the difference between $r$ and $\bar{r}$ is small, it carries a new piece of physics: the fact that the guiding-centres deviate from a magnetic field line by a few Larmor radii (a banana width at most). This radial excursion leads to a parallel flow.

The impact of the initial distribution function on the dynamics of the equilibrium $\mathbf{E} \times \mathbf{B}$ flow $\left\langle v_{\theta}\right\rangle$ has been analysed numerically. A set of two simulations is compared in Fig. 1: they only differ by the initial distribution function, either given by the canonical (Eq. (8)) or by the non-canonical (Eq. (1)) expression. As evident in Fig. 1a, the system develops a large mean flow $\phi_{00}$ on short time scales when initialised with the non-canonical distribution function. It turns out to be more than one order of magnitude larger than the one observed in the canonical case. The poloidal cross-section of the electric potential then exhibits the annular structure characteristic of such large scale flows. In this non-canonical case, it is worth noticing that the system also develops spontaneously a parallel flow that tends to counter balance the vertical charge separation. However, such a process appears to build up on larger time scales. The quantitative dynamics of both the electric field generation and the parallel flow will be addressed in a future publication. Ultimately, one may expect the large equilibrium flow appearing in the non-canonical case to possibly reduce the turbulence magnitude. Following earlier theoretical works [9-11], an approximate criterion to quantify the backreaction of the velocity shear on the turbulence is provided by the ratio of the $\mathbf{E} \times \mathbf{B}$ shearing rate $\gamma_{E}=\left|\partial_{r r} \phi_{00} / B\right|$ over the linear growth rate $\gamma_{\text {lin }}$. The latter is approximated by $\gamma_{\text {lin }} \approx\left(k_{\theta} \rho_{i}\right) v_{\mathrm{T}} / \sqrt{R_{0} L_{\mathrm{p}}}$ in Fig. $1 \mathrm{~b}$, with $k_{\theta} \rho_{i} \approx 0.3$ corresponding to the most unstable linear mode [1], $v_{\mathrm{T}}=\sqrt{T / m}$ the thermal velocity and $L_{\mathrm{p}}$ the equilibrium pressure gradient length. According to this criterion, the non-canonical case appears to be marginally unstable, $\gamma_{E} / \gamma_{\text {lin }}$ being of the order of 1, while the canonical case remains unstable until later times. Fig. 1c focuses on the time evolution of the most unstable resonant mode in both canonical and non-canonical simulations. In the canonical case this mode grows, whereas it remains at the initial perturbation level in the non-canonical case, possibly being stabilised by the $\mathbf{E} \times \mathbf{B}$ shear.

Such an analysis remains qualitative, since both the stability criterion and the linear growth rate are approximations. To sum up, it essentially shows that (i) the non-canonical initialisation leads indeed to the
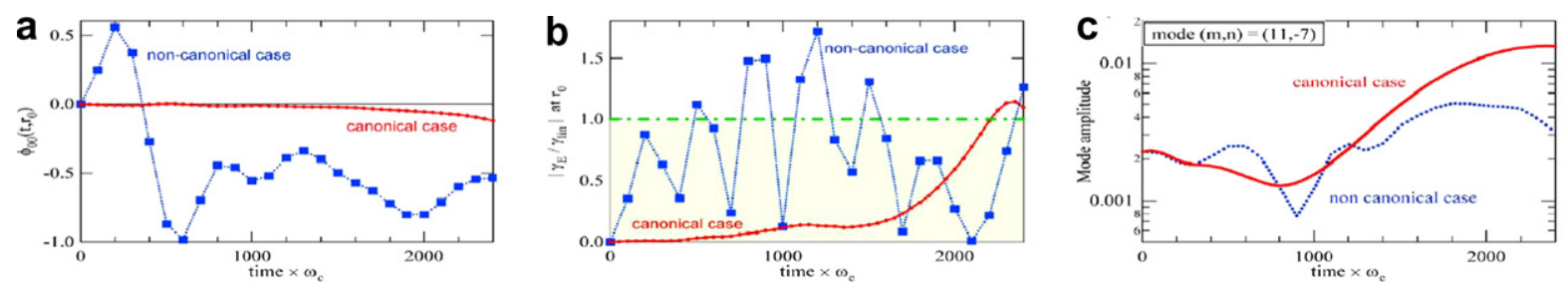

Fig. 1. In both canonical and non-canonical cases $\left(\rho^{\text {औ }}=10^{-2}\right)$ : (a) time evolution of the $(m, n)=(0,0)$ mode of the electric potential (b) ratio of the $\mathbf{E} \times \mathbf{B}$ shearing rate over a rough estimate of the linear growth rate (c) time evolution of the most unstable mode. 
fast generation of large scale sheared $\mathbf{E} \times \mathbf{B}$ flows, and that (ii) these flows can potentially reduce the effective instability growth rate. If strong enough, such flows could reduce and possibly quench the turbulence.

\section{Consistency with the force balance equation}

The consistency between fluid and gyrokinetic descriptions is a matter of current work, as exemplified in Ref. [15] in the general gyrokinetic-MHD framework. The present section focuses on some aspects of this consistency, restricted to the simplified equations solved by the Gysela code. Namely, we only consider the calculation restricted to the first-order in $\rho^{\star}$, consistently with the latter reference. Let us first notice that the specific prescription for the equilibrium distribution function adopted in Eq. (9), including the definition of $\bar{v}_{\|}$, belongs to the following larger class of equilibrium distribution functions:

$$
f_{\text {eq }}=\frac{n(\bar{\psi})}{(2 \pi T(\bar{\psi}) / m)^{3 / 2}} \exp \left(-\frac{\mathscr{H}}{T(\bar{\psi})}+\frac{e \phi(\bar{\psi})}{T(\bar{\psi})}\right)\left\{1+\frac{m \bar{w}_{\|} \bar{\kappa}}{T(\bar{\psi})}\right\}
$$

Here, $\bar{\psi}$ is derived from the third invariant: $\bar{\psi} \equiv \frac{P_{\varphi}}{e}=\psi+\frac{m R v_{\varphi}}{e}, \psi$ being the poloidal flux. $\bar{\psi}$ is analogous to $\bar{r}$ in that it represents an effective radial coordinate in the case of a more general class of axisymmetric magnetic equilibria of the form: $\mathbf{B}=I \nabla \varphi+\nabla \psi \times \nabla \varphi$. Here, $I$ is a flux function $I(\psi)$. The density and temperature profiles in Eq. (1) are now evaluated with $\bar{\psi}$, and thus are motion invariants. Also, $\bar{w}_{\|}$and $\bar{\kappa}$ are arbitrary functions of the motion invariants. They are such that $\frac{m \bar{w} \| \bar{k}}{T(\bar{\psi})}$ scales like $\rho^{\star}$.

This section aims at showing the simple relationship between $\bar{\kappa}$ and fluid quantities such that Gysela fulfills the force balance equation. Let us first detail the force balance in fluid:

$$
e n(\mathbf{E}+\mathbf{V} \times \mathbf{B})=\nabla p
$$

Summing over all species, this equation implies $\mathbf{B} \cdot \nabla p=0$ and $\mathbf{E} \cdot \mathbf{B}=-\mathbf{B} \cdot \boldsymbol{\nabla} \phi=0$, meaning that the pressure $p$ and the electrostatic potential $\phi$ are flux functions. The general solution of Eq. (12) reads

$$
\mathbf{V}=\mathbf{V}_{E}+\mathbf{V}^{\star}+V_{\|} \frac{\mathbf{B}}{B}=-\nabla \psi \times \frac{\mathbf{B}}{B^{2}}\left(\phi^{\prime}+\frac{p^{\prime}}{n e}\right)+V_{\|} \frac{\mathbf{B}}{B}
$$

where $p^{\prime}$ and $\phi^{\prime}$ stand for the derivatives of $p$ and $\phi$ with respect to $\psi$. Uppercase letter $\mathbf{V}$ refers to fluid velocities. The cross-product can be derived from the general expression of the magnetic field: $\nabla \psi \times \mathbf{B}=I \mathbf{B}-B^{2} R^{2} \nabla \varphi$. Let us define $\frac{\kappa_{\mathrm{f}}}{B_{\max }(\psi)} \equiv \frac{-I}{B^{2}}\left(\phi^{\prime}+\frac{p^{\prime}}{n e}\right)+\frac{V_{\|}}{B}$. With these new notations, the fluid velocity $\mathbf{V}$ (Eq. (13)) reads as follows:

$$
\mathbf{V}=\frac{\kappa_{f}}{B_{\max }(\psi)} \mathbf{B}+\left(\phi^{\prime}+\frac{p^{\prime}}{n e}\right) R^{2} \nabla \varphi
$$

where $B_{\max }$ is the maximum magnetic field on each magnetic surface: $B_{\max }(\psi) \equiv B(\psi, \theta=\pi)$. Fig. 2 illustrates the different ways of expressing the fluid velocity $\mathbf{V}$ : field aligned and transverse coordinates are used in Eq. (13), whereas Eq. (14) expresses $\mathbf{V}$ as a difference between two adjacent directions, namely $\boldsymbol{\nabla} \varphi$ and $\mathbf{B}$. In this latter system of coordinates, the match between the fluid and the gyrokinetic approaches is easily tackled.

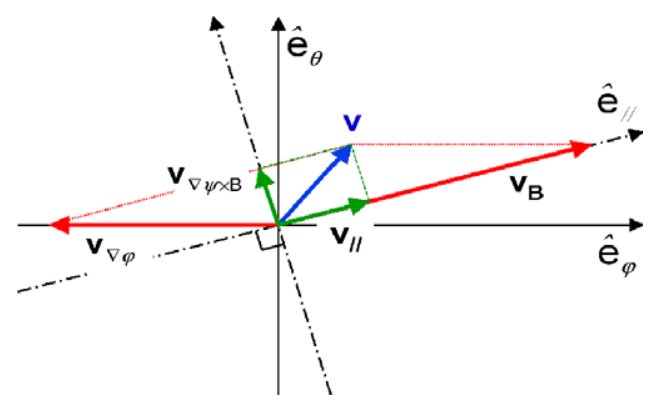

Fig. 2. In flux coordinates, the field lines (given by $\hat{\mathbf{e}}_{\|}$) are straight lines. 
In the limit of large aspect ratios, incompressibility can be reasonably assumed: $\boldsymbol{\nabla} \cdot \mathbf{V}=0$. In this case, $\mathbf{B} \cdot \nabla \frac{\kappa_{f}}{B_{\max }}=0$, such that $\kappa_{\mathrm{f}}$ is a flux function $\kappa_{\mathrm{f}}(\psi)$. The parallel and transverse components of $\mathbf{V}$ then read:

$$
\begin{aligned}
& V_{\|}=\kappa_{f}(\psi) \frac{B}{B_{\max }(\bar{\psi})}+\left(\phi^{\prime}+\frac{p^{\prime}}{n e}\right) \frac{I(\psi)}{B} \\
& \mathbf{V}_{\perp}=\mathbf{V}_{E}+\mathbf{V}^{\text {出}}=\mathbf{V}_{E}+\frac{\mathbf{B} \times \mathbf{\nabla} p}{e n B^{2}}
\end{aligned}
$$

Let us now express the fluid velocity as derived from the general form for the kinetic equilibrium in GysELA, Eq. (11). The standard definitions for the parallel and transverse guiding-center fluid velocities of such an equilibrium are $\mathscr{V}_{\|}=\frac{1}{n} \int v_{\|} f_{\text {eq }}$ and $\mathscr{V}_{\perp}=\frac{1}{n} \int\left(\mathbf{v}_{g}+\mathbf{v}_{E}\right) f_{\text {eq }}$, where integration is carried out over normalised phase space $\int \equiv \int_{0}^{\infty} 2 \pi B / m \mathrm{~d} \mu \int_{-\infty}^{+\infty} \mathrm{d} v_{\|}$. It is easy to show that both kinetic and fluid transverse flows exhibit the same divergence. Indeed, let us first recall that $\int e \mathbf{v}_{g} \cdot \nabla f_{\mathrm{eq}}=\frac{\mathbf{B}}{B^{2}} \times \frac{\mathrm{\nabla} B}{B} \cdot \nabla\left[\int\left(m v_{\|}^{2}+\mu B\right) f_{\mathrm{eq}}\right]$. The MHD equilibrium imposes $\mathbf{j} \times \mathbf{B}=\nabla p$, such that the later term can be recast into $2 \frac{\mathbf{B} \times \mathbf{\nabla} B}{B^{3}} \cdot \nabla p=\nabla \cdot\left(\frac{\mathbf{B} \times \nabla p}{B^{2}}\right)$. The divergence of $\mathscr{V}_{\perp}$ then reads: $\nabla \cdot \mathscr{V}_{\perp}=\boldsymbol{\nabla} \cdot\left(\mathbf{V}_{E}+\frac{\mathbf{B} \times \nabla p}{e n B^{2}}\right)$. One recovers both the electric drift and the magnetising current obtained in the fluid description. Up to a rotational term, $\mathscr{V}_{\perp}$ and $\mathbf{V}_{\perp}$ are then equal. The calculation of the magnetising current from the transverse particle fluid velocity is given in Appendix A.

As far as the parallel flow is concerned, first notice that the difference between $\bar{\psi}$ and $\psi$, namely $m R v_{\varphi} / e$, remains small and scales like $\rho^{\star}$. One can then Taylor expand $f_{\text {eq }}$, Eq. (11):

$$
f_{\mathrm{eq}}(\bar{\psi}) \simeq f_{\mathrm{eq}}(\psi)+\left.(\bar{\psi}-\psi) \frac{\mathrm{d} f_{\mathrm{eq}}}{\mathrm{d} \bar{\psi}}\right|_{\psi} \simeq f_{\mathrm{M}}(\psi)+f_{\mathrm{M}}(\psi) \frac{m w_{\|} \kappa}{T(\psi)}+f_{\mathrm{M}}(\psi) \frac{m I v_{\|}}{e}\left\{\frac{n^{\prime}}{n}+\frac{T^{\prime}}{T}\left[\frac{E}{T}-\frac{3}{2}\right]+\frac{e \phi^{\prime}}{T}\right\}
$$

$w_{\|}$and $\kappa$ correspond to $\bar{w}_{\|}(\bar{\psi})$ and $\bar{\kappa}(\bar{\psi})$ where $\bar{\psi}$ is replaced by $\psi$, respectively. $f_{\mathrm{M}}$ is defined by Eq. $(1) . f_{\mathrm{M}}(\psi)$ does not lead to any parallel flow. The two last terms only of Eq. (17) contribute to $\mathscr{V}_{\|}$:

$$
\mathscr{V}_{\|}=\frac{m \kappa}{T}\left\langle\left\langle v_{\|} w_{\|}\right\rangle\right\rangle+\frac{I(\psi)}{B}\left(\phi^{\prime}+\frac{p^{\prime}}{n e}\right)
$$

where $\langle\langle\mathscr{G}\rangle\rangle \equiv \frac{1}{n} \int_{0}^{\infty} 2 \pi B / m \mathrm{~d} \mu \int_{-\infty}^{+\infty} \mathrm{d} v_{\|} \mathscr{G} f_{\mathrm{M}}(\psi)$. The parallel velocities given in Eqs. (18) and (15) look similar. Giving $\bar{w}_{\|}$a similar expression to that of $\bar{v}_{\|}$(Eq. (10)), namely:

$$
\bar{w}_{\|}=\operatorname{Sign}\left(v_{\|}\right) \sqrt{\frac{2}{m}} \sqrt{E-\mu B_{\max }(\bar{\psi})} \mathscr{Y}\left(E-\mu B_{\max }(\bar{\psi})\right)
$$

with $\mathscr{Y}$ the Heaviside function, one obtains $\frac{m \kappa}{T}\left\langle\left\langle v_{\|} w_{\|}\right\rangle\right\rangle=\kappa_{f} \frac{B}{B_{\max }}$. In this case, $\mathbf{V}_{\|}$and $\mathscr{V}_{\|}$can be reconciled provided $\kappa=\kappa_{f}$, or equivalently $\bar{\kappa}=\kappa_{f}(\bar{\psi})$.

\section{Conclusion}

This paper provides a practical way to tackle the delicate problem of the equilibrium in $f u l l-f$ and collisionless gyrokinetic codes such as GYSELA 5D, aiming at predicting the turbulent transport level in tokamak plasmas. Especially, a large scale electric field is found to develop on short time scales if the initial distribution function is not an equilibrium function - for instance a Maxwellian with constant profiles on magnetic surfaces. This field results from the imbalanced vertical charge separation which naturally occurs in such toroidal configurations. This large scale electric potential leads to a sheared poloidal rotation, which back reacts on turbulence and can potentially quench the instability, if large enough. Conversely, choosing a canonical initial distribution function, i.e. a stationary equilibrium of the gyrokinetic equation, cures such a problem. The canonical form retained in GYSELA 5D, function of the motion invariants only, aims at minimising the difference between an effective radius based on the invariants, and the geometrical radius. It is shown to fulfill the force balance equation.

\section{Appendix A. Recovering the particle transverse fluid current}

We shall here emphasise on the classical result which enables to derive the correct particle transverse fluid velocity. It is well known that the transverse current $\mathbf{J}_{\perp}$ reads: $\mathbf{J}_{\perp}=\mathbf{J}_{\perp \mathrm{gc}}+\mathbf{J}_{\text {magn }}$, where $J_{\perp \mathrm{gc}}$ is the guiding-center 
component, $J_{\text {magn }}=\boldsymbol{\nabla} \times \mathbf{M}$ is the magnetisation current, $\mathbf{M}=-\int \mu \mathbf{b} f$ the plasma magnetisation and integration is carried out over normalised phase space $\int \equiv \int_{0}^{\infty} 2 \pi B / m \mathrm{~d} \mu \int_{-\infty}^{+\infty} \mathrm{d} v_{\|}$. The following calculation is performed taking into account the 'low $\beta$-drift', namely $\mathbf{v}_{D}=\mathbf{v}_{g}+\frac{m v_{\|}^{2}}{e B^{2}}(\boldsymbol{\nabla} \times \mathbf{B})_{\perp}$.

$$
\begin{aligned}
\mathbf{J}_{\perp} & =\int e\left(\mathbf{v}_{E}+\mathbf{v}_{D}\right) f_{\mathrm{eq}}+(\boldsymbol{\nabla} \times \mathbf{M})_{\perp}=e n \mathbf{V}_{E}+p \frac{\mathbf{B} \times \boldsymbol{\nabla} B}{B^{3}}+\frac{(\boldsymbol{\nabla} \times \mathbf{B})_{\perp}}{B^{2}} \int m v_{\|}^{2} f_{\mathrm{eq}}-\left(\boldsymbol{\nabla} \times \int \mu \mathbf{b} f_{\mathrm{eq}}\right)_{\perp} \\
& =e n \mathbf{V}_{E}+\frac{\mathbf{B} \times \boldsymbol{\nabla} p_{\perp}}{B^{2}}+\left(p-2 p_{\perp}\right) \frac{\mathbf{B} \times \boldsymbol{\nabla} B}{B^{3}}+\left(p_{\|}-p_{\perp}\right) \frac{(\boldsymbol{\nabla} \times \mathbf{B})_{\perp}}{B^{2}}
\end{aligned}
$$

which is the transverse fluid current up to the first-order in $\rho^{\text {tr }}$, the pressure being defined as follows: $p_{\|} \equiv \int m v_{\|}^{2} f_{\mathrm{eq}}, p_{\perp} \equiv \int \mu B f_{\mathrm{eq}}$ and $p=p_{\|}+p_{\perp}$. In the case of an isotropic pressure tensor, $p_{\|}=p_{\perp}$, the last two terms vanish and the expression for $\nabla \cdot \mathscr{V}_{\perp}$ is recovered.

\section{References}

[1] Dimits AM, Bateman G, Beer MA, Cohen BI, Dorland W, Hammett GW, et al. Comparisons and physics basis of tokamak transport models and turbulence simulations. Phys Plasmas 2000;7(3):969-83.

[2] Politzer PA. Observation of avalanchelike phenomena in a magnetically confined plasma. Phys Rev Lett 2000;84(6):1192-5. February.

[3] Carreras BA, Newman D, Lynch VE, Diamond PH. A model realization of self-organized criticality for plasma confinement. Phys Plasmas 1996;3(8):2903-11.

[4] Garbet X, Sarazin Y, Beyer P, Ghendrih P, Waltz RE, Ottaviani M, et al. Flux driven turbulence in tokamaks. Nucl Fusion 1999;39:2063-8.

[5] Beyer P, Benkadda S, Garbet X, Diamond PH. Nondiffusive transport in tokamaks: three-dimensional structure of bursts and the role of zonal flows. Phys Rev Lett 2000;85(23):4892-5.

[6] Grandgirard V, Brunetti M, Bertrand P, Besse N, Garbet X, Ghendrih Ph, et al. A drift-kinetic semi-lagrangian 4d code for ion turbulence simulation. J Comput Phys 2006;217(2):395-423.

[7] Grandgirard V, Sarazin Y, Garbet X, Dif-Pradalier G, Ghendrih Ph, Crouseilles N, et al. Transport and large scale flows in ion temperature gradient turbulence. Commun Nonlinear Sci Numer Simul 2008;13 [on-line July 2007].

[8] Garbet X, Sarazin Y, Grandgirard V, Dif-Pradalier G, Darmet G, Ghendrih Ph, Bertrand P, Besse N, Gravier E, Morel P, Sonnendrucker E, Crouseilles N, Dischler JM, Latu G, Violard E, Brunetti M, Brunner S, Lapillonne X, Tran TM, Villard L, Boulet M. Beyond scale separation in gyrokinetic turbulence. In: Proceedings of the 21st IAEA fusion energy conference, Chengdu 2006, Nucl Fusion [submitted for publication].

[9] Biglari H, Diamond PH, Terry PW. Influence of sheared poloidal rotation on edge turbulence. Phys Fluids B: Plasma Phys 1990;2(1):1-4.

[10] Waltz RE, Kerbel GD. Advances in the simulation of toroidal gyro-landau fluid model turbulence. Phys Plasmas 1995;2(6):2408-16.

[11] Hahm TS, Burrell KH. $E \times b$ flow shear effects on radial correlation length of turbulence and gyroradius scaling of confinement. Phys Plasmas 1996;3(1):427-9.

[12] Hazeltine RD, Meiss JD. Plasma confinement. Addison-Wesley; 1992.

[13] Idomura Y, Tokuda S, Kishimoto Y. Global gyrokinetic simulation of ion temperature gradient driven turbulence in plasmas using a canonical maxwellian distribution. Nucl Fusion 2003;43(4):April.

[14] Angelino P, Bottino A, Hatzky R, Jolliet S, Sauter O, Tran TM, et al. On the definition of a kinetic equilibrium in global gyrokinetic simulations. Phys Plasmas 2006;13(5):052304.

[15] Qin H, Tang WM, Rewoldt G, Lee WW. On the gyrokinetic equilibrium. Phys Plasmas 2000;7(3):991-1000. 